Crime, Histoire \& Sociétés / Crime, History \& Societies

Vol. 21, n² | 2017

L'histoire de la criminalité et de la justice pénale : propositions de recherche pour le $21^{\mathrm{e}}$ siècle

\title{
Une justice transitionnelle en chantier : le cas du Rwanda
}

\section{Ornella Rovetta}

\section{(2) OpenEdition Journals}

Édition électronique

URL : http://journals.openedition.org/chs/1908

DOI : $10.4000 /$ chs. 1908

ISSN : 1663-4837

Éditeur

Librairie Droz

Édition imprimée

Date de publication : 31 décembre 2017

Pagination : 143-153

ISSN : $1422-0857$

Référence électronique

Ornella Rovetta, "Une justice transitionnelle en chantier : le cas du Rwanda », Crime, Histoire \& Sociétés / Crime, History \& Societies [En ligne], Vol. 21, n² | 2017, mis en ligne le 19 juillet 2020, consulté le 13 janvier 2021. URL : http://journals.openedition.org/chs/1908 ; DOl : https://doi.org/10.4000/chs.1908 


\title{
Une justice transitionnelle en chantier: le cas du Rwanda
}

\author{
Ornella Rovetta
}

Zin décembre 1996 débutaient, devant les tribunaux de première instance rwandais, les premiers procès organisés sur la base d'une nouvelle loi sur le génocide et les crimes contre l'humanité adoptée quelques mois plus tôt. Cette loi encadre le jugement «des personnes poursuivies d'avoir, à partir du 1eroctobre 1990, commis des actes qualifiés et sanctionnés par le Code pénal et qui constituent: soit des crimes de génocide ou des crimes contre l'humanité [...], soit des infractions visées au Code pénal qui, selon ce qu'allègue le ministère Public ou admet l'accusé, ont été commises en relation avec les événements entourant le génocide et les crimes contre l'humanité ${ }^{1}$. Une semaine plus tard, un premier procès international s'ouvrait à Arusha, en Tanzanie, devant le Tribunal pénal international pour le Rwanda (TPIR). L'accusé, un ancien bourgmestre, comparaissait pour génocide, incitation directe et publique à commettre le génocide, crimes contre l'humanité et violations de l'article 3 commun aux Conventions de Genève de 1949. Le Tribunal international devait en particulier juger les responsables se trouvant hors du territoire rwandais et ayant fait partie des autorités politiques, militaires, médiatiques et économiques. Quelques années plus tard, en avril 2001, 110journalistes couvraient le début du procès de ceux qu'on a appelés «les quatre de Butare» devant la cour d'assises de Bruxelles ${ }^{2}$. C'est la loi de compétence dite «universelle» adoptée en 1993 qui a permis de poursuivre deux religieuses, un professeur d'université et un directeur d'usine, tous Rwandais. Pour l'une des avocates des parties civiles à l'époque, ce procès montre que «le monde se rétrécit autour des criminels contre l'humanité ${ }^{3}$. En 2006, après une phase-pilote et une phase de récolte de données débutée en 2002, les milliers de tribunaux locaux gacaca installés aux niveaux des cellules et des secteurs au Rwanda commencèrent à juger de très nombreux citoyens rwandais. À Paris, enfin, s'est tenu en 2014, un procès en cour d'assises dans la cause d'un ex-officier de la garde présidentielle.

Loi organique sur l'organisation des poursuites des infractions constitutives du crime de génocide ou de crimes contre l'humanité, commises à partir du $1^{\text {er }}$ octobre 1990, nº 8/96, Kigali, République de Rwanda, 1996.

2 Borloo, J.P. (2001) Un épisode du génocide jugé à Bruxelle, Le Soir, [En ligne] http://www.lesoir. be/archives/recup\%2525Fcrimes-de-guerre-la-belgique-innove-en-confiant-au-jury_t-20010414Z0KCDX.html (consulté le 9 novembre 2017).

3 Déclaration de Maître Michèle Hirsch, «Extrait de l'acte de partie civile», cour d'assises de l'arrondissement administratif de Bruxelles-Capitale, procès de Vincent Ntezimana, Alphonse Higaniro, Consolata Mukangango, et Julienne Mukabutera, avril 2001. En ligne: http://assisesrwanda2001. org/040200.html (consulté le 23 octobre 2017). 
Si la «justice transitionnelle» est cet objet mal défini qui englobe «une large palette de moyens juridiques organisant la confrontation avec les méfaits des régimes antérieurs dans des périodes de changement politique $»^{4}$, l'histoire du Rwanda ces vingt-trois dernières années apparaît comme un cas d'école qui permet d'examiner ce concept sur deux plans: la durée de la justice transitionnelle et l'imbrication de différentes compétences juridiques. Le processus judiciaire qui s'ouvre avec le début quasi simultané des procès devant les tribunaux de première instance rwandais et devant le TPIR fin 1996 s'inscrit en effet dans un temps judiciaire post-génocide long qui n'a, aujourd'hui, pris fin qu'en partie. Du point de vue de l'étendue du champ juridique, la poursuite des crimes de génocide perpétrés au Rwanda d'avril à juillet 1994 a nécessité de repenser les différentes échelles de justice et de définir de nouvelles compétences, du local à l'international. Ainsi, aux deux extrémités du spectre judiciaire, le TPIR créé par le Conseil de sécurité des Nations unies le 8 novembre 1994 et les tribunaux gacaca instaurés au Rwanda en 2001 sont connectés tant par les faits dont ils sont saisis - tous les procès portent sur les massacres du printemps 1994 - que par les terrains d'enquête qui se croisent à travers la recherche des preuves et des témoins.

Le jugement des crimes de génocide commis au Rwanda contre les Tutsi en 1994 a conduit à près de vingt ans de procès internationaux, dix ans de procès au niveau micro-local, et, parce qu'il s'agit de crimes imprescriptibles, il est possible que de nouveaux procès devant les tribunaux rwandais et étrangers soient organisés dans les années à venir. En 1945, l'intense phase de poursuite menée par les Alliés s'est achevée au début des années 1950, suivie d'une période où de grands procès (comme les procès Eichmann, Barbie, Touvier ou celui de Francfort) ont été organisés de manière plus espacée. Au Rwanda, les vingt années qui ont suivi le génocide ont été marquées par un nombre croissant et continu de procès à différentes échelles. Mais quelle est la durée d'une transition? Quand s'arrête la transition? Cet article propose une réflexion sur l'articulation entre justice transitionnelle et écriture historique. Il interroge les implications méthodologiques du travail sur les «archives transitionnelles», en partant du cas du Rwanda.

\section{INTERROGER LES RECONFIGURATIONS}

Dans les «nouvelles après-guerres» telles que définies par la philosophe Isabelle Delpla pour l'ex-Yougoslavie, la justice est articulée aux échelles locale, nationale et internationale et doit jouer un rôle politique et social central ${ }^{5}$. La place accordée par les acteurs politiques (comme résultat d'une victoire militaire ou d'une transition négociée) à ceux qu'ils entendent juger et la manière dont ce processus doit influer sur le monde social (par des programmes de «réconciliation» par exemple) est donc caractéristique des politiques transitionnelles, dont la mise en place répond à la fois à «un agenda politique et juridique » ${ }^{6}$. Mais dans ces contextes singuliers de «l'après », Isabelle Delpla montre aussi qu'on tend à considérer comme après-guerre ce que

Traduction par l'auteure de l'anglais. Définition de Ruti Teitel citée par Priemel (2013, p. 553-554).

Delpla (2014, p. 21-22).

Huyse (2014, p.352). 
l'on observe à travers le prisme des politiques de justice ou des programmes de reconstruction ${ }^{7}$. La justice transitionnelle n'est donc pas seulement un outil qui agit sur et dans les espaces observés, mais elle interagit aussi avec le regard du chercheur dans l'étude de ces processus et dans l'utilisation du matériau qui en résulte.

Or, si l'historiographie a montré comment les méthodes du juge et de l'historien diffèrent dans leur objet et leur objectif, la saisie du passé est précisément l'un des points de rencontre entre le droit et l'histoire ${ }^{8}$. Dans les après-guerres où la justice pénale nationale et internationale a joué un rôle important, celle-ci a produit ses effets au-delà des espaces mis en place pour gérer la transition. Le processus d'écriture historique peut ainsi s'engager à l'intérieur et à l'extérieur des prétoires, de manière simultanée ou différée, en se fondant ou non sur les archives qui résultent de ces mesures transitionnelles. L'impact des sources produites par les procès de Nuremberg en 1945-1948 sur l'historiographie de la Seconde Guerre mondiale ou le rôle des historiens dans l'élaboration de l'acte d'accusation dans le cadre du procès de Francfort de 1963-1965, illustrent bien ces dynamiques ${ }^{9}$. Pour pouvoir décrypter les faits jugés, il faut donc accéder à la grammaire des décisions judiciaires, et, plus largement, aux dynamiques des mécanismes de justice transitionnelle. Une attention pour les acteurs et les pratiques des institutions transitionnelles permet alors de déconstruire le cadre de l'énoncé, c'est-à-dire le prétoire, l'enquête judiciaire, ou la commission vérité et réconciliation ${ }^{10}$.

\section{TEMPORALITÉ DE LA TRANSITION}

La temporalité très courte dans laquelle intervient le débat, durant l'été 1994 sur la pluralité des réponses judiciaires à apporter au génocide contre les Tutsi, puis l'adaptation progressive du cadre juridique national dans les années suivantes ne sont pas sans rappeler les choix effectués après la Seconde Guerre mondiale. Là aussi, la question de la justice s'est posée pour la première fois durant la guerre ${ }^{11}$. Elle a donné lieu à l'élaboration d'un cadre juridique international, puis de législations nationales. Au Rwanda, la période de transition vers un nouveau gouvernement et les échanges qui s'engagent et surtout s'intensifient durant l'été 1994, dans l'immédiat aprèsgénocide, montrent comment différents aspects qui sont théorisés comme relevant de la justice transitionnelle, sont discutés : réconciliation et rejet de l'amnistie, séparation entre procès nationaux et internationaux, mise en œuvre concrète de la justice, rapatriement des réfugiés et question des détenus dont le nombre s'accroît rapidement dans les prisons rwandaises. À la même époque, la transition en Afrique du Sud conduira à un mécanisme tout autre et emblématique des politiques transitionnelles: la commission vérité et réconciliation.

Delpla (2014, p. 21-22).

Thomas (1998, p. 17-36); Ginzburg (1997).

Bloxham (2001); Wittmann (2005).

10 Sur le cadre énonciatif des témoignages au Tribunal pénal international pour l'ex-Yougoslavie, voir Claverie (2012, p. 169-210).

11 Smith (1981); Weisers (2014); Brayard (2000). 
Les politiques de la transition sont, historiquement, traversées par la dichotomie d'un choix entre recours ou exclusion de pratiques judiciaires classiques pour gérer le passé. La «justice transitionnelle» tente, elle, depuis les années 1980, de concilier justice et politique, de «réconcilier justice et paix » ${ }^{12}$. Au Rwanda, face à l'ampleur et à la rapidité avec laquelle près d'un million de personnes ont été massacrées en trois mois, il a fallu inventer de nouveaux modes de confrontation avec ce passé ${ }^{13}$. La voie judiciaire vers la «réconciliation nationale» a été largement privilégiée ${ }^{14}$. Ce choix a entraîné un processus transitionnel long, marqué par l'adoption de différentes lois et par une juxtaposition des cadres juridiques, mais aussi par une porosité dans la division du travail judiciaire. À titre d'exemple, des élus locaux ont été jugés devant les tribunaux de première instance rwandais, le TPIR, les juridictions gacaca et la cour d'assises de Paris.

Les politistes et juristes ont développé une approche comparative principalement fondée sur le cadre juridique des différentes cours saisies du contentieux de génocide, plutôt que sur les procès ${ }^{15}$. Mais ces nouvelles juridictions ont donné lieu à une vaste jurisprudence qui joue également un rôle central pour l'écriture de l'histoire. Ces espaces de justice ont en effet été investis par les historiens comme lieux de production de sources. Les procès ont mis en lumière les enjeux d'une histoire critique des identités dites «ethniques», d'une analyse des modes de participation aux massacres et de déconstruction de l'idéologie politique; autant de thèmes pour lesquels les historiens ont été interrogés par la justice - comme experts et témoins et donc, comme acteurs du processus judiciaire. C'est particulièrement vrai pour le TPIR et pour les procès devant les cours de pays tiers qui ont organisé des procès, comme la Suisse, la Belgique, l'Allemagne ou la France.

Sur un autre plan, l'échec de la communauté internationale face au génocide des Tutsi et le débat persistant et extrêmement sensible sur le rôle de la France au Rwanda avant et en 1994 ont aussi conduit à l'organisation d'une série de commissions d'enquête parlementaires et d'experts. Le Rapport de la commission indépendante d'enquête sur les actions de l'Organisation des Nations unies lors du génocide de 1994 au Rwanda de 1999 a constitué un mea culpa de la communauté internationale, tout comme le rapport de la commission parlementaire belge de 1997 qui a pointé de nombreuses lacunes dans l'appréciation de la situation par les autorités belges ${ }^{16}$. En 2000, l'Organisation de l'Unité africaine se situait dans la même veine avec un rapport intitulé Rwanda: un génocide qu'on aurait pu éviter. Mais les travaux de la mission d'information française de 1998 sur les opérations militaires menées par la France au Rwanda entre 1990 et 1994 et le rapport d'une commission indépendante rwandaise sur l'implication de l'État français durant la même période (rapport de la Commission Mucyo du nom de l'ancien ministre rwandais de la Justice) sont loin

\footnotetext{
12 Lefranc (2009, p. 565).
}

13 Le dénombrement réalisé par le gouvernement rwandais recense 1074017 victimes déclarées et 934218 victimes dénombrées. République du Rwanda, ministère de l'Administration locale, du Développement communautaire et des Affaires sociales, Dénombrement des victimes du génocide. Rapport final (version révisée), Kigali, avril 2004 ; l'ONU retient le chiffre de plus de 800000 victimes.

14 Dumas (2008, p. 112).

15 Voir notamment Palmer (2015); Magnarella (2000); Jones (2010).

16 Voir notamment les trois analyses suivantes: Le Pape et al. (1999). 
d'avoir établi un récit partagé et d'avoir résolu les zones d'ombre ${ }^{17}$. La publication récente dans la revue $X X I$ d'un article mettant en cause directement les autorités françaises dans le réarmement des forces gouvernementales rwandaises en fuite en juillet 1994 (et la non-divulgation des archives relatives à ces faits), ainsi que la plainte déposée contre BNP-Paribas pour financement d'armes en violation de l'embargo en soulignent l'actualité brûlante ${ }^{18}$.

\section{ENTRE TRANSITION ET HISTOIRE}

La vingtième commémoration du génocide contre les Tutsi rwandais en 2014 s'est inscrite dans une transformation progressive du champ de la justice postgénocide, avec la fin des procès devant les juridictions gacaca en 2012, puis du TPIR en 2015 et le procès en France en 2014 d'un ex-officier de la garde présidentielle rwandaise ${ }^{19}$. La fin de ces processus constitue un point de rupture institutionnel qui transforme le statut des archives qui en résultent. Il est remarquable que, comme pour le Tribunal pénal international pour l'ex-Yougoslavie, les travaux du TPIR ont été accessibles aux chercheurs en temps réel. La publicité des débats, par la mise en ligne des transcriptions in extenso des audiences a permis d'avoir accès à des milliers de témoignages entendus devant cette cour. On peut bien entendu mettre des bémols à cette ouverture, notamment parce qu'il ne s'agit que des pièces publiques de la phase du procès et non des éléments d'enquête, des témoignages à huis clos et des éléments de preuve couverts par un régime de confidentialité. Mais les bases de données d'archives des tribunaux ad hoc créées dans les années 1990 constituent néanmoins un moyen unique d'accéder à la richesse de cette documentation ${ }^{20}$.

Le rôle central de cette documentation était déjà manifeste dans l'un des premiers ouvrages de référence sur le génocide publié en 1999 par Alison Des Forges, Human Rights Watch et la Fédération internationale des droits de l'homme. Dans Aucun témoin ne doit survivre, les archives du TPIR jouent en effet un rôle important dans l'analyse du génocide à l'échelon national et local, au même titre que les dossiers judiciaires rwandais et les sources belges produites dans le cadre des enquêtes menées par le juge d'instruction Damien Vandermeersch dès 1995. L'ouvrage conclut cette première appréhension des faits à différentes échelles par une réflexion sur les mécanismes de justice mis en place après le génocide et soutient que seule une justice individuelle, mais aussi «d'autres mécanismes de manifestation de la vérité»

17 Audoin-Rouzeau (2010, p. 122-134).

18 Saint-Exupéry, P. de (2017) Réarmez-les, XXI, 39; Malagardis, M. (2017) Rwanda: la BNP visée par une plainte pour complicité de génocide, Libération, Paris.

19 Je m'appuie ici sur une réflexion menée à l'occasion d'une présentation conjointe: Marcel Kabanda et Ornella Rovetta «L'écriture de l'histoire du génocide des Tutsi: les sources et les leçons du prétoire», université libre de Bruxelles, workshop Conflits, Mémoire et Justice. Perspectives de recherche sur les Grands Lacs africains, 9 juin 2017.

20 Les archives judiciaires publiques du TPIR ont migré sur différentes plateformes avant d'être rassemblées dans la nouvelle base de données créée au moment de la fermeture du TPIR et de la fin des travaux du TPIY. Archives du mécanisme pour les tribunaux pénaux internationaux, base de données des archives judiciaires en ligne [http://jrad.unmict.org] (consulté le 27 octobre 2017). 
pourront produire un récit accepté et acceptable pour les Rwandais ${ }^{21}$.

On le sait, la justice agit profondément sur notre compréhension des faits et sur leur mémoire ${ }^{22}$. L'accès en quasi-temps réel à une partie des sources judiciaires, et la possibilité d'observer ces processus qui se sont déroulés au Rwanda, à Arusha, à Bruxelles ou à Lausanne, pose donc des défis à la recherche sur une justice transitionnelle ${ }^{23}$.

L'imprescriptibilité des crimes internationaux actée par la Convention de 1968 sur les crimes de guerre et les crimes contre l'humanité et reconnue par de nombreuses législations nationales, a prolongé le temps de la justice. Dans cette configuration, justice, mémoire et histoire s'articulent et interagissent. Des séquences de justice transitionnelle peuvent alors être définies et étudiées, comme le montre l'historien Nico Wouters pour l'Europe des années 1940, 1970 et 199024. Dans le projet de recherche collectif Jusinbellgium «Un siècle de jurisprudence pionnière. Une base de données digitales de précédents belges en matière de justice internationale, 19142014 », nous avons pour ambition de décaler ce regard pour y intégrer une histoire critique de la justice après le premier conflit mondial à travers le cas de la Belgique ${ }^{25}$. L'échec d'un tribunal international, la tentative des procès de Leipzig en 1921 et l'organisation méconnue de procès par défaut de militaires allemands accusés de violations des lois et coutumes de la guerre en 1924-1925 devant les conseils de guerre belges participent des tâtonnements dans la mise en œuvre d'une «nouvelle justice $»^{26}$. L'analyse de ces mécanismes par un travail sur les archives des enquêtes et des procès permet d'interroger cet événement dans une perspective historique plus large. La poursuite des inciviques, la mise en place de tribunaux administratifs pour les dommages de guerre et l'exclusion de fonctionnaires ayant servi l'ennemi sont autant de décisions qui relèvent d'ailleurs, pour Jos Monballyu, d'une «justice transitionnelle» qui n'existe bien sûr pas en tant que telle dans la décennie qui suit la fin de la Première Guerre mondiale ${ }^{27}$. Il y a donc une tension entre la conceptualisation de la «justice transitionnelle» dans les années 1990 et son application à des contextes qui précèdent sa création comme concept analytique ${ }^{28}$. Les deux après-guerres en Belgique ont donné lieu à des politiques de poursuite de la collaboration, un passé qui, singulièrement pour la Seconde Guerre mondiale reste un enjeu sociétal important, notamment en ce qui concerne l'accès aux archives ${ }^{29}$. La comparaison historique des réponses relevant de ce qu'on appelle «justice transitionnelle» aux crimes

\footnotetext{
21 Des Forges (1999, p. 855).

22 Rousso (1990, p. 186-187).

23. Cet article se fonde en large partie sur ma recherche doctorale: Rovetta (2013).

24 Wouters (2013, p. 369-411).

25 Je fais ici référence à mes recherches menées dans le cadre du projet Jusinbellgium avec mes collègues Pieter Lagrou, Delphine Lauwers, Hendrik Vandekerckhove, Wolfgang Form et Marie-Anne Weisers. Je remercie Marie-Anne Weisers pour la relecture de cet article et pour ses remarques et suggestions. Voir pour plus d'informations sur ce projet financé par le programme Brain de Belspo: http://jusinbell.hypotheses.org

26 Lewis (2014).

27 Monballyu (2012, p. 443-479).

28 Voir pour la Seconde Guerre mondiale Priemel et Stiller (2012).

29 Rousseaux et van Ypersele (2008); Aerts et al. (2017).
} 
commis à grande échelle et contre des populations civiles ouvre ainsi la possibilité de cartographier la justice post-conflit ${ }^{30}$. Dans cette perspective, les politiques judiciaires après des épisodes de violence de masse font partie de l'histoire de l'événement en tant que tel.

\section{RÉCEPTION(S) ET TRANSMISSION}

La question de la réconciliation et de la reconstruction de la société rwandaise après un génocide "populaire $»^{31}$ et un «génocide au village " $^{32}$ qui a impliqué un nombre très élevé d'auteurs et de coauteurs à tous les échelons de la société, occupe une place centrale dans la littérature transitionnelle. Retenue dans la résolution qui a créé le TPIR en novembre 1994, la notion de «réconciliation» recherchée à travers les processus de justice et la recherche de la vérité n'en demeure pas moins un concept flou et pluriel. Il est aussi présent au niveau institutionnel rwandais avec la création en 1999 de la National Unity and Reconciliation Commission. Au-delà des discours qui s'articulent autour de l'unité nationale et de la réconciliation et dont on comprend l'objectif dans un pays détruit par la guerre et le génocide, la question de la réception des politiques de justice demeure sans doute l'un des axes d'investigation les plus complexes.

Comment les catégories juridiques influent-elles sur la place des victimes dans ces processus? Quelle est la part active des victimes à titre collectif ou individuel dans la justice transitionnelle? Les recherches actuelles offrent de nouvelles manières d'appréhender ces mécanismes car elles mettent en lumière non seulement leur cadrage institutionnel, mais aussi leur fabrication plus fine à travers les réseaux associatifs par exemple. Le cas de l'Amérique latine depuis le milieu des années 1970 est emblématique d'une voix des victimes qui réclame la vérité sur les dictatures au Chili ou en Argentine; une demande qui se développe en dehors de tout cadre judiciaire, pour, ensuite, dans les années 1990 venir contester les amnisties mises en place et réclamer une réponse pénale aux violations massives des droits de l'homme ${ }^{33}$. Dans ces contextes, les réseaux d'associations et les militants des droits de l'homme sont des acteurs importants de la documentation des faits. C'est aussi sur ce type d'acteurs que la justice transitionnelle s'est appuyée au Rwanda et en ex-Yougoslavie, même si la circulation des expertises et des expériences ne doit pas masquer les spécificités de chaque après-conflit. Au-delà des associations, les individus - témoins et victimes jouent un rôle qui est infléchi non seulement par les règles du prétoire, mais aussi par le contexte social et politique du témoignage devant les juridictions internationales. Ce contexte peut conduire à la volonté de reconnaissance devant les tribunaux, ou au

30 Cet article s'appuie aussi sur ma participation à la «Mission d'étude en France sur la recherche et l'enseignement des génocides et des crimes de masse» qui a tenu ses travaux sous la direction de Vincent Duclert d'octobre 2016 à octobre 2017, et dont les analyses et les études de cas discutées ont considérablement enrichi ma connaissance de ces mécanismes.

31 Kimonyo (2008).

32 Dumas $(2014,2015)$.

33 Naftali (2015, p 139). 
contraire au refus de témoigner ${ }^{34}$. L'identité, la langue, la posture du témoin sont ainsi des points d'attention cruciaux pour la compréhension des procès ayant pour objet les crimes de masse comme le montre Christian Delage dans son analyse du cas du témoin et survivant de la Shoah Abraham Sutzkever au procès de Nuremberg en $1946^{35}$.

Les associations de survivants comme Ibuka au Rwanda, en Belgique et en France sont, comme d'autres avant elles, actives dans le domaine de la justice, du niveau micro-local au prétoire international. Ils sont des acteurs incontournables de la justice transitionnelle et de la mémoire des faits. Dans un processus que l'historien Henry Rousso appelle la «mondialisation de la mémoire ${ }^{36}$, les expériences de la gestion du passé nazi et de la Shoah, ses formes de mobilisation et ses demandes de justice jouent un rôle important dans la configuration des sociétés post-conflit contemporaines.

Les sources judiciaires semblent presque incontournables dans l'étude du génocide contre les Tutsi rwandais tant elles documentent la diversité de cet événement historique. Comme toute source historique, elles ne peuvent toutefois parler que si on les interroge. L'histoire locale du génocide les mobilise en les croisant le plus souvent avec un travail d'entretiens avec les rescapés et les auteurs des crimes. Ce faisant, ce travail de reconstitution pose en filigrane la question de la réception des processus de justice transitionnelle et de leur articulation avec les récits individuels. Au Rwanda, les expériences des survivants tutsi et des génocidaires ont été souvent exposées à une médiation du langage judiciaire, qu'il soit local - dans les gacaca - ou international - au TPIR. Mais étudier le déploiement de la violence nécessite parfois pour le chercheur de "rompre avec la forme du procès $»^{37}$, son langage, son fonctionnement, ses logiques. L'attention pour cette multiplicité des médiations permet aussi d'interroger la capacité des victimes et des témoins à agir sur le processus de justice et à examiner l'articulation entre mémoire et justice ${ }^{38}$.

Avec la fin des procès internationaux et des tribunaux gacaca-les premiers ayant laissé une vaste collection documentaire déjà accessible en large partie, les seconds étant en cours de numérisation - s'ouvre un nouvel épisode en termes d'écriture historique. L'année 2014 a sans doute constitué un tournant dans la clôture d'une «première » période transitionnelle de vingt ans. Loin de clore le débat sur la mémoire et la justice, celle-ci a aussi été marquée par une urgence de l'histoire face aux discours d'inintelligibilité produits sur le génocide contre les Tutsi ${ }^{39}$. Des historiens que l'on pourrait qualifier d'engagés, ont, pour contrer les discours sur une haine «ancestrale» qui aurait provoqué une violence imprévisible et insensée, tenté d'analyser l'événement à travers des articles scientifiques, mais aussi par l'expertise apportée dans les tribunaux. Parallèlement, les politiques transitionnelles dans le contexte rwandais ont, bien que de manière imparfaite, établi les responsabilités individuelles. Soixante-quatorze personnes jugées par le TPIR (dont une décédée avant la fin de son procès), des dizaines dans les pays tiers, et 818564 suspects identifiés en 2006

\footnotetext{
34 Jouhanneau (2016); Stover (2005).

35 Delage (2010).

36 Voir le chapitre «Une mondialisation de la mémoire» dans Rousso (2016, p. 275).

37 Baraduc (2014, p. 64).

38 Voir les recherches en cours de Rasmont $(2016,2008)$.

39 Chrétien (1995, p. 131-142).
} 
et 1958634 dossiers finalement traités par les tribunaux gacaca ${ }^{40}$ au Rwanda: les sources résultant de ces processus ne constituent qu'une part de l'histoire, mais elles sont centrales pour comprendre à la fois les faits et la manière dont ils ont été jugés.

Ornella Rovetta

Université libre de Bruxelles orovetta@ulb.ac.be

\section{BIBLIOGRAPHIE}

Aerts, K., Luyten, D., Willems, B., Drossens, P. et Lagrou, P. (2017) Papy était-il un nazi? Sur les traces d'un passé de guerre, Bruxelles: Racine.

Audoin-Rouzeau, S. (2010) La responsabilité de la France vue du Rwanda. Le rapport Mucyo: une lecture historienne, Esprit, dossier France-Rwanda, et maintenant ?, 364, p. 122-134.

Baraduc, V. (2014) Tuer au cœur de la famille. Les femmes en relais Vingtième Siècle. Revue d'histoire, 122, 2.

Bloxham, D. (2001) Genocide on trial: war crimes trials and the formation of Holocaust history and memory, Oxford, New York: Oxford University Press.

Brayard, F. (Dir.) (2000), Le génocide des Juifs entre procès et histoire, 1943-2000, Bruxelles: Éditions Complexes.

Brehm, H.N., Uggen, C., Gasanabo, J.-D. (2014) Genocide, justice and Rwanda's gacaca courts, Journal of Contemporary Criminal Justice, 30, 3, p. 333-352.

Chrétien, J.-P. (1995) Un nazisme tropical au Rwanda? Images ou logique d'un génocide, Vingtième Siècle, 48, 1, p. 131-142, [En ligne] DOI: 10.2307/3770219.

Claverie, E. (2012) Démasquer la guerre: chronique d'un nettoyage ethnique, Visegrad (BosnieHerzégovine), printemps 1992, L'Homme, 3, p. 169-210.

Delage, C. (2010) La place du témoin filmé. De Nuremberg au procès des Khmers rouges, Le Débat, 158, p.38-41, [En ligne] DOI: 10.3917/deba.158.0032.

Delpla, I. (2014) La justice des gens: enquête dans la Bosnie des nouvelles après-guerre, Rennes: Presses universitaires de Rennes.

Des Forges, A. (1999) Aucun témoin ne doit survivre: le génocide au Rwanda, Paris: Karthala.

Dumas, H. (2008) Histoire, justice et réconciliation: les juridictions gacaca au Rwanda, Mouvements, 53, 1, p. 110-117.

Dumas, H. (2014) Le génocide au village. Le massacre des Tutsi au Rwanda, Paris: Seuil.

Dumas, H. (2015), Rwanda : comment juger un génocide ?, Politique étrangère, 4, p. 39-50, [En ligne] DOI: 10.3917/pe.154.0039.

Ginzburg, C. (1997) Le juge et l'historien: considérations en marge du procès Sofri, Lagrasse: Verdier (pour la traduction française).

Huyse, L. (2014) Comparing transitional justice experiences in Europe, in Wouters, N. (Ed.), Transitional justice and memory in Europe (1945-2013), Cambridge: Intersentia Publishing.

40 Rutayisire (2012, p. 51), Brehm et al. (2014). 
Jones, N.A., (2010) The courts of genocide: Politics and the Rule of Law in Rwanda and Arusha, Abingdon \& New York: Routledge.

Jouhanneau, C. (2016) Sortir de la guerre en Bosnie-Herzégovine. Une sociologie politique du témoignage et de la civilité, Paris: Karthala.

Kimonyo, J.-P. (2008) Rwanda, un génocide populaire, Paris: Karthala.

Le Pape, M., Willame, J.-C. et Chrétien, J.-P. (1999) Débat: Rwanda. Réflexions sur les rapports parlementaires de la Belgique et de la France, Politique africaine, 73, 1, p. 159176, [En ligne] DOI: 10.3917/polaf.073.0159.

Lefranc, S. (2009) La professionalisation d'un militantisme réformateur du droit: l'invention de la justice transitionnelle, Droit et société, 73, 3, p.561-589.

Lewis, M. (2014) The Birth of the New Justice: The Internationalization of Crime and Punishment, 1919-1950, Oxford: Oxford University Press.

Magnarella, P.J. (2000) Justice in Africa: Rwanda's genocide, its courts, and the UN criminal tribunal, Aldershot \& Brookfied Vt.: Ashgate.

Monballyu, J. (2012) La justice transitionnelle en Belgique dans les affaires pénales après la Première Guerre mondiale (1918-1928), Revue d'Histoire du Droit, 80, p.443-479, [En ligne] DOI: 10.1163/15718190-000A1216.

Naftali, P. (2015) Le droit à la vérité à l'épreuve de ses mobilisations en Amérique latine: entre ressource et contrainte, Revue interdisciplinaire d'études juridiques, 75, 2, [En ligne] DOI: 10.3917/riej.075.0139.

Palmer, N. (2015) Courts in Conflict: Interpreting the Layers of Justice in Post-genocide Rwanda, Oxford, New York: Oxford University Press.

Priemel, K.C. (2013) Consigning justice to history: transitional trials after the Second World War, The Historical Journal, 56, 2, p. 553-581.

Priemel, K.C. et Stiller A. (2012) Reassessing the Nuremberg Military Tribunals: Transitional Justice, Trial Narratives and Historiography, New York: Berghahn.

Rasmont, F. (2008) Histoire, mémoire et génocide: l'asbl «Ibuka-Mémoire et justice» de juillet 1994 à nos jours (mémoire en histoire, université libre de Bruxelles).

Rasmont, F. (2016) La mémoire du génocide tutsi : une porte d'entrée pour explorer la sociabilité rwandaise pré et post-génocide, Contemporanea, 3, [En ligne] http://contemporanea.be/nl/ article/2016-3-review-fr-rasmont (consulté le 13 novembre 2017).

Rousseaux, X et van Ypersele, L. (2008) La patrie crie vengeance: La répression des «inciviques » belges au sortir de la guerre 1914-1918, Bruxelles: Éditions Le Cri.

Rousso, H. (1990) Le syndrome de Vichy: de 1944 à nos jours (2 édition revue et augmentée), Paris: Seuil.

Rousso, H. (2016) Face au passé: Essais sur la mémoire contemporaine, Paris: Belin (Collection Histoire).

Rovetta, O. (2013) Le Tribunal pénal international pour le Rwanda comme source d'histoire? (thèse de doctorat, université libre de Bruxelles).

Rutayisire, P. (Ed.) (2012) Evaluation of Gacaca process: Achieved results per objective (Centre for Conflict Management).

Smith, B.F. (1981) The Road to Nuremberg, New York: Basic Books.

Stover, E. (2005) The Witnesses: War crimes and the promise of justice in The Hague, Philadelphia: University of Pennsylvania Press. 
Thomas, Y. (1998) La vérité, le temps, le juge et l'historien, Le Débat, 5, 102, p. 17-36.

Weisers, M.-A. (2014) Juger les crimes contre les Juifs : des Allemands devant les tribunaux belges, 1941-1951 (thèse de doctorat, université libre de Bruxelles).

Wittmann, R. (2005) Beyond justice: the Auschwitz trial, Cambridge, Mass: Harvard University Press.

Wouters, N. (2013) Transitional Justice and Memory Development in Europe, in Wouters, N. (Ed.) Transitional justice and memory in Europe (1945-2013), Cambridge: Intersentia Publishing, p. 369-411. 